\title{
An Experimental Study on the Capability test of a Gas leakage checker for Extinguishing Agent Vessels
}

\author{
JiHyun Kwark ${ }^{1, *}$, SungHo Hong ${ }^{1}$, MoonSoo Choi ${ }^{1}$, DongSuk Kim ${ }^{1}$ and GoSeop Roh ${ }^{2}$ \\ ${ }^{1}$ Fire Insurers Laboratories of Korea, 1030 Gyeongchung-daero, Ganam-eup, Yeoju-si, Gyeonggi-do, 12661, Korea \\ ${ }^{2}$ Jinhwa Engineering \& Construction, 12, Bangbaecheon-ro 4-gil, Seocho-gu, Seoul, Korea \\ *Corresponding author
}

\begin{abstract}
In this study the capability test of newly developed gas leakage checkers for gaseous extinguishing systems was conducted. The leakage checker consists of valve body, slip nut, plunger and micro switch etc. Until now extinguishing agent leakage has been checked through direct measuring with a level gauge by a man or measuring its weight using a load cell. The network based leakage checkers for a gaseous fire extinguishing system are able to supervise any gas leakage or discharge for real time 24/7. The working pressure was measured for both leakage checkers and the capability test was repeated 3 times respectively. The averaged working pressure of both leakage checkers were 0.31 bar and 0.30 bar respectively. Consequently the capability of both leakage checkers appeared available.
\end{abstract}

Keywords-capability test; gaseous extinguishing system; gas leakage checker; extinguishing agent vessel

\section{INTRODUCTION}

The agent vessels of a gaseous fire extinguishing system are ready to leak extinguishing agent because of ageing and superannuating of vessel's valve. According to the National Fire Safety Code (NFSC) a leaked vessel shall be refilled or exchanged if the loss of agent excesses $5 \%$. Until now agent leakage of a vessel has been checked through direct measurement with a level gauge by a man or measuring its weight using a load cell; however an ordinary supervision system for agent leakage has not fully developed yet.

In this study network based leakage checkers for a gaseous fire extinguishing system were developed. The leakage checkers are able to supervise any gas leakage or discharge for real time 24/7. The capability of two kinds of leakage checkers was verified by experiment.

\section{ELEMENT DESIGN AND DEVELOPMENT OF A GAS LEAKAGE CHECKER}

In this study 2 kinds of gas leakage checkers were developed to supervise any leakage or discharge from the vessel of HFC clean extinguishing agent i.e. HFC-23 and HFC-227ea.

They are based on network system and able to give a notice promptly as soon as any leakage occurred. Each leakage checker consists of valve body, slip nut, plunger and micro switch. Figure I shows the 3D drawing of both leakage checkers.

They were produced for use of an agent vessel with 68 liters volume. The compressed pressure of agent is $4.2 \mathrm{MPa}$ at $25^{\circ} \mathrm{C}$, the nominal size of main valve is $40 \mathrm{~A}$ and its screw size is $21 / 4$ inches.

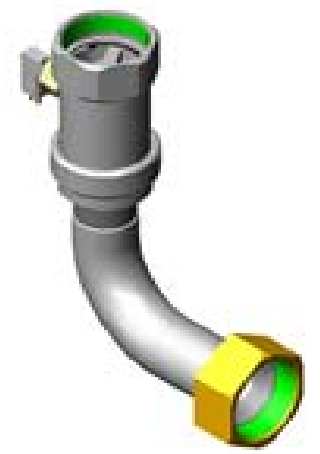

(a) HFC-23

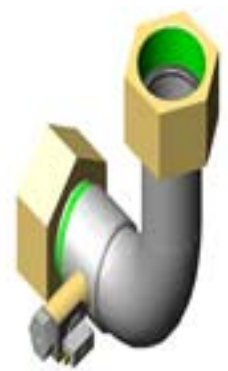

(b) HFC-227ea
Figure I. 3D DRAWING OF LEAKAGE CHECKERS

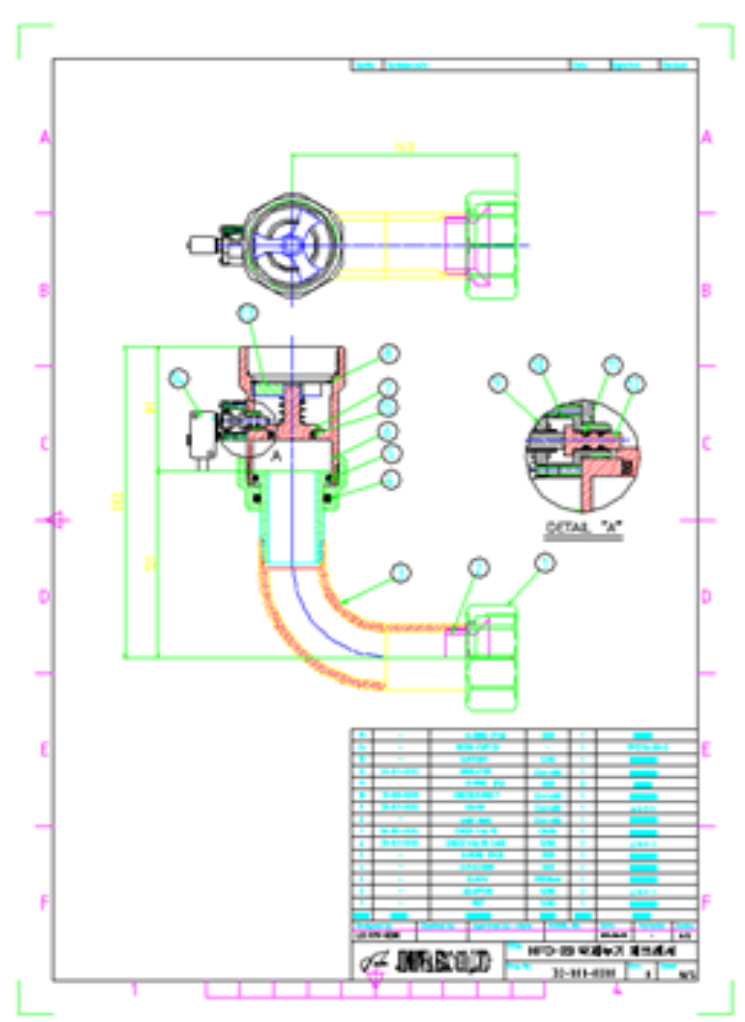

Figure II. LAYOUT OF THE LEAKAGE CHECKER FOR HFC-23 


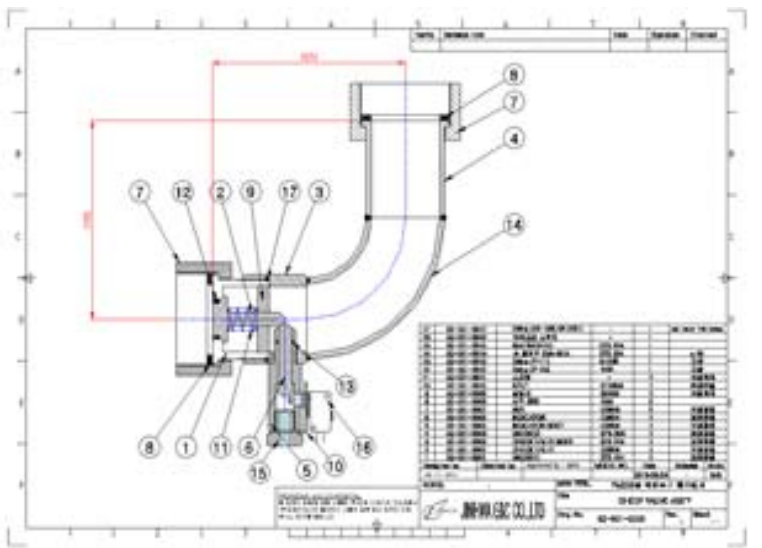

Figure III. LAYOUT OF THE LEAKAGE CHECKER FOR HFC-227EA

TABLE I. SPECIFICATION OF ELEMENT DESIGN

\begin{tabular}{|c|c|}
\hline Item & Specification \\
\hline Outlet size & $40 \mathrm{~A}($ nominal) \\
\hline Min. working pressure & 0.3 bar \\
\hline Switch & Micro switch(On/Off) \\
\hline Screw & $21 / 4$ inches \\
\hline Material & C3771BE \\
\hline
\end{tabular}

III. EXPERIMENTAL APPARATUS AND METHOD

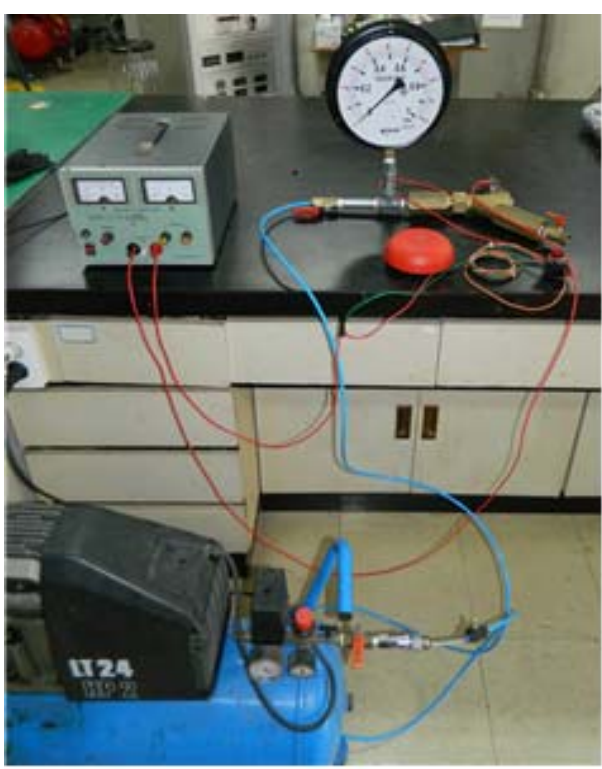

Figure IV. EXPERIMENTAL APPARATUS

As shown in Figure IV the experimental apparatus used to check gas leakage consists of leakage checker, pressure gauge, metal tee, power supply, bell(ramp) and air compressor. The pressure gauge was installed on the gas leakage checker using the metal tee and the air compressor was connected to the opposite side of the metal tee.
Electric power was supplied to the leakage checker as shown in Figure $\mathrm{V}$ and also it was connected to the bell and the ramp to make a sensing signal.

With increase of air pressure the leakage checker begins to work and the bell rings when accumulated pressure inside the leakage checker reaches the limit 0.3 bar.

The working pressure was measured for both leakage checkers and the capability test was repeated 3 times respectively.

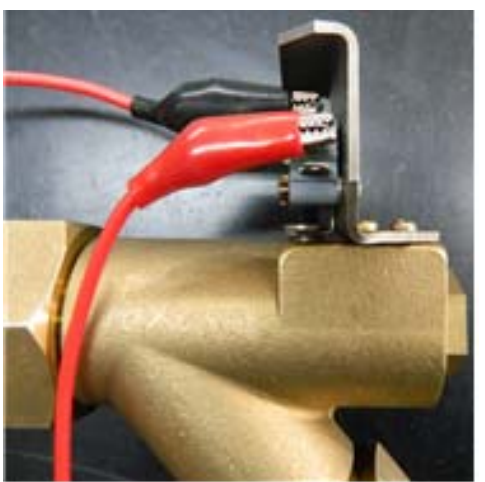

Figure V. DETAILS OF POWER CONNECTING ON THE LEAKAGE CHECKER

\section{EXPERIMENTAL RESULTS AND CONSIDERATIONS}

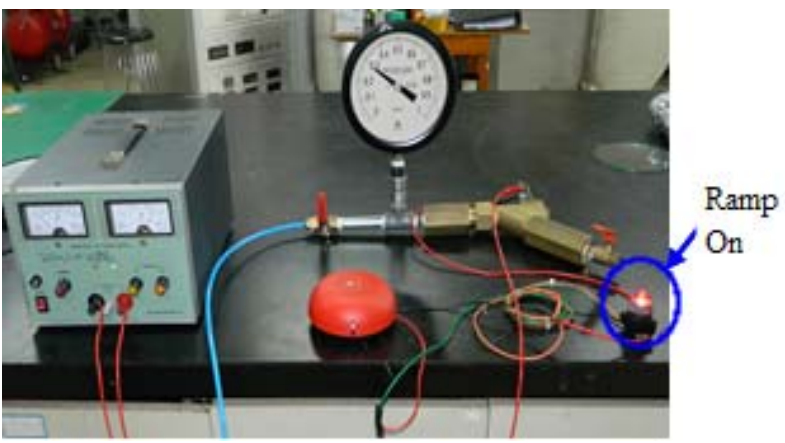

Figure VI. SENSING MOMENT OF GAS LEAKAGE NEAR 0.3 BAR

In the test of both leakage checkers working pressure appeared nearly constant as shown in Table II. With increase of air pressure the leakage checkers initially didn't show any reaction. However when air pressure reached around the designed working pressure 0.3 bar, the leakage checkers worked well by ringing and blinking. The averaged working pressure of both leakage checkers were 0.31 bar and 0.30 bar respectively. Consequently the capability of both leakage checkers appeared available.

TABLE II. EXPERIMENTAL RESULTS

\begin{tabular}{|c|c|c|c|c|}
\hline \multirow{2}{*}{$\begin{array}{c}\text { Leakage } \\
\text { checker }\end{array}$} & \multicolumn{4}{|c|}{ Working(sensing) pressure(bar) } \\
\cline { 2 - 5 } & 1st & 2nd & 3rd & Average \\
\hline HFC-23 & 0.31 & 0.32 & 0.31 & 0.31 \\
\hline HFC-227ea & 0.30 & 0.31 & 0.29 & 0.30 \\
\hline
\end{tabular}




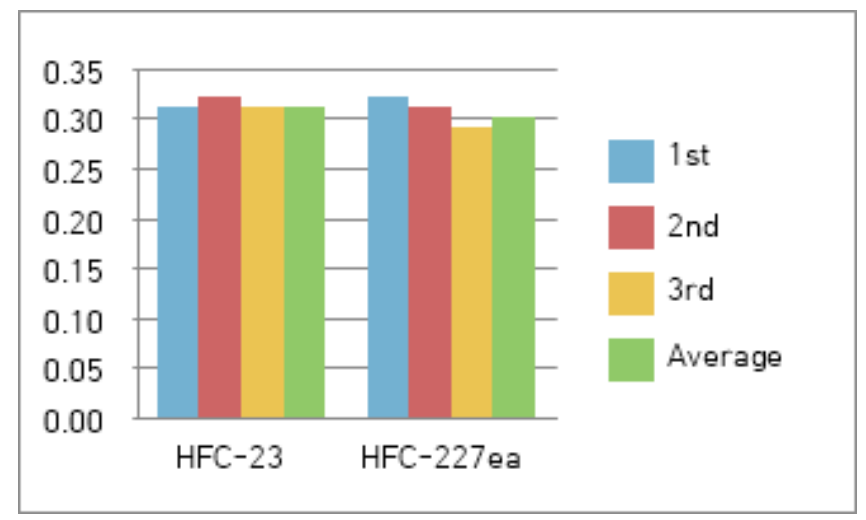

Figure VII. EXPERIMENTAL RESULTS OF BOTH LEAKAGE CHECKERS

\section{CONCLUSION}

In this study the capability of network based gas leakage checkers was verified by experiment. The gas leakage checker for extinguishing agent vessels was designed to supervise any gas leaking for $24 / 7$ by sensing accumulated gas pressure when the leaked gas pressure inside the checker reaches the critical value. If we can know the leaking state of the agent vessels for real time, the fire extinguishing performance and credibility of extinguishing systems will be able to increase.

In the near future the sensing characteristics of the leakage checkers will be analyzed in accordance with leaking flow rate and also various resistant tests will be conducted according to environmental conditions.

\section{ACKNOWLEDGMENT}

This research was supported by the National Emergency Management Agency (2014-NEMA14-029-01020000-2014).

\section{REFERENCES}

[1] J. H. Ku, "A Study on Personal Safety Improvement of the Gaseous Fire Extinguishing System”, Journal of Korean Society Hazard Mitigation, Vol.14, No.3, pp. 193-199, June 2014

[2] J. I. Yoon, J. H. Choi, "A Development of Methodology for NOVEC Gas Fire Extinguishing System", Journal of the Korean Society of Marine Engineering, Vol.39, No.3, pp. 206-210, March 2015

[3] FM 3010, Approval Standard for Fire Alarm Signaling Systems, December 2010

[4] B. S. Son, H. W. Kim, "A Study on Percent Agent in Pipe as a Criterion to Evaluate Limitations and Performance of Gaseous Fire Extinguishing Systems", Journal of Korean Institute of Fire Science \& Engineering, Vol.21, No.4, pp 1-11, December 2007

[5] C. H. Yang, "Applying examples of sensors in the automatic systems", Monthly Automatic Control, 2002

[6] ISO 7240-2, Fire detection and alarm systems -- Part 2: Control and indicating equipment, September 2003 